\title{
Open versus percutaneous tracheostomy in COVID-19: a multicentre comparison and recommendation for future resource utilisation
}

\author{
Aleix Rovira ${ }^{1}$ - Stephen Tricklebank ${ }^{2} \cdot$ Pavol Surda $^{1} \cdot$ Stephen Whebell ${ }^{2}$ Joe Zhang ${ }^{2} \cdot$ Arun Takhar $^{1}$. \\ Elizabeth Yeung ${ }^{3} \cdot$ Kathleen Fan $^{3} \cdot$ Imran Ahmed $^{4} \cdot$ Phillip Hopkins $^{5} \cdot$ Deborah Dawson $^{6} \cdot$ Jonathan Ball $^{6}$. \\ Ram Kumar ${ }^{7}$. Waqas Khaliq ${ }^{8} \cdot$ Ricard Simo $^{1} \cdot$ Asit Arora $^{1}$
}

Received: 10 November 2020 / Accepted: 24 December 2020 / Published online: 9 January 2021

(c) Crown 2021

\begin{abstract}
Purpose The COVID-19 pandemic placed an unprecedented demand on critical care services for the provision of mechanical ventilation. Tracheostomy formation facilitates liberation from mechanical ventilation with advantages for both the patient and wider critical care resource, and can be performed using both percutaneous dilatational and surgical techniques. We compared outcomes in those patients undergoing percutaneous dilatational tracheostomy to those undergoing surgical tracheostomy and make recommendations for provision of tracheostomy services in any future surge.

Methods Multicentre multidisciplinary retrospective observational cohort study including 201 patients with COVID-19 pneumonitis admitted to an ICU in one of five NHS Trusts within the South London Adult Critical Care Network who required mechanical ventilation and subsequent tracheostomy.

Results Percutaneous dilatational tracheostomy was performed in 124 (62\%) of patients, and surgical tracheostomy in 77 $(38 \%)$ of patients. There was no difference between percutaneous dilatational tracheostomy and surgical tracheostomy in either the rate of peri-operative complications (16.9 vs. $22.1 \%, p=0.46)$, median [IQR(range)] time to decannulation [19.0 (15.0-30.2 (5.0-65.0)] vs. 21.0 [15.5-36.0 (5.0-70.0) days] or mortality $(13.7 \%$ vs. $15.6 \%, p=0.84)$. Of the 172 patients that were alive at follow-up, two remained ventilated and 163 were decannulated.

Conclusion In patients with COVID-19 pneumonitis that require tracheostomy to facilitate weaning from mechanical ventilation, there was no difference in outcomes between those patients that had percutaneous dilatational tracheostomy compared with those that had surgical tracheostomy. Planning for future surges in COVID-19-related critical care demands should utilise all available resource and expertise.
\end{abstract}

Keywords Covid-19 $\cdot$ Percutaneous tracheostomy $\cdot$ Surgical tracheostomy $\cdot$ Mechanical ventilation

\section{Introduction}

The COVID-19 pandemic placed an unprecedented global demand on Critical Care services. In the UK, approximately 13,000 patients required admission to Critical Care, many of

Aleix Rovira and Stephen Tricklebanck equally contributed as first authors.

Supplementary Information The online version contains supplementary material available at https://doi.org/10.1007/s0040 5-020-06597-1.

Aleix Rovira

aleix.rovira@gstt.nhs.uk

Extended author information available on the last page of the article whom required advanced respiratory support [1]. Not only does liberation from mechanical ventilation represent a crucial step towards survival for critically ill patients, it also has clear resource implications where demand for mechanical ventilators is high. Tracheostomy formation is a gold standard intervention for patients where mechanical ventilation is anticipated to be prolonged and is commonly used to facilitate weaning [2-5].

Tracheostomy can be performed using both percutaneous dilatational (PDT) and surgical (ST) techniques. Complication rates are comparable, although some reports suggest lower rates of minor bleeding and wound infection with PDT compared to ST [6-15].

The COVID-19 pandemic necessitated the rapid development of new collaborative, multidisciplinary ways of 
providing patient care, utilising all available resources [16]. Five of our acute hospitals in South London responded to the unprecedented increase in demand for tracheostomy in a variety of ways, depending on individual resource availability. This report describes our experience with tracheostomy during the COVID-19 pandemic, compares outcomes in those patients undergoing PDT compared to ST, and based on this makes recommendations for provision of tracheostomy services in any future surge.

\section{Methods}

This is a multicentre retrospective observational cohort study, conducted between 1st March and 5th May 2020 in accordance with the strengthening the reporting of observational studies in epidemiology (STROBE) statement. All data were routinely recorded contemporaneously to either and then analysed retrospectively, to evaluate the impact of tracheostomy technique on patients with respiratory failure requiring mechanical ventilation and subsequent tracheostomy for anticipated prolonged respiratory wean. We did not include patients that received extracorporeal membrane oxygenation (ECMO) during their admission. Using the NHS Health Research Authority decision tool, this project was determined to be a service evaluation thereby not requiring ethical approval, and was registered with the audit departments of each participating hospital. Principles of Good Clinical Practice were adhered to throughout the study.

Patients were admitted to a critical care unit in one of five NHS Trusts within the South London Adult Critical Care Network: Guy's and St Thomas' NHS Foundation Trust, Kings College Hospital NHS Foundation Trust, Lewisham and Greenwich NHS Trust, Kingston Hospital NHS Foundation Trust and St George's University Hospitals NHS Foundation Trust. As part of the South London operational delivery network surge response, additional critical care capacity was created at Guy's and St Thomas' NHS Foundation Trust, Kings College Hospital NHS Foundation Trust and St George's University Hospitals NHS Foundation Trust, and patients included those transferred between units for capacity reasons. All patients had severe acute respiratory syndrome coronavirus-2 (SARS-CoV-2) infection confirmed with reverse transcriptase polymerase chain reaction (RTPCR) of nasopharyngeal, oropharyngeal or tracheal samples.

Data obtained included baseline demographic, laboratory and physiological characteristics. Acute Physiology and Chronic Health Evaluation (APACHE II) scores at critical care admission were recorded where available, as well as ventilation parameters from initiation of ventilation, until $24 \mathrm{~h}$ following tracheostomy. Key events in each patient's admission were noted, including tracheostomy-related complications, liberation from mechanical ventilation, tracheostomy decannulation and death. The end point for mechanical ventilation was defined as when the patient tolerated ventilator-free breathing for at least $24 \mathrm{~h}$. Patients were followed up until death, tracheostomy decannulation (whichever occurred first) or to a maximum of 110 days. Where inter-hospital transfer and subsequent repatriation occurred for capacity reasons, patients were cross-checked to avoid duplication and data combined. All data were managed and analysed in Python (packages: NumPy 1.19.0, SciPy v1.4.1). Continuous quantitative data did not uniformly follow normal distribution and are presented with mean (SD) or medians (IQR[range]), with comparison between groups using Mann-Whitney $U$. Categorical variables are presented as frequencies and percentages and compared using Fisher's exact test or Pearson's Chi-squared test.

\section{Tracheostomy technique and decision-making}

The decision to perform tracheostomy was made jointly by two critical care consultants after evaluation of clinical course and prognosis. Choice of technique, operator and equipment was based primarily on local resource and expertise in individual hospitals, but patient factors including body habitus, adequacy of neck extension and grade of laryngoscopy were also considered. PDT was performed at the bedside on the ICU by either an otorhinolaryngologist or oral maxillofacial surgeon following a period of structured training [17], or a critical care clinician. ST was performed either in theatre or at the bedside by either an otorhinolaryngologist or an oral maxillofacial surgeon. All procedures were performed wearing appropriate personal protective equipment. Weaning from mechanical ventilation was managed by the critical care multidisciplinary team. Decannulation was managed by the tracheostomy multidisciplinary team and took place either on the ICU or on the ward.

\section{Peri-operative complications}

Peri-operative complications were recorded if they were considered to be a direct result of the tracheostomy procedure, were clinically significant or required intervention, and they occurred either during the procedure ("intra-operative complication") or within seven days of the procedure ("post-operative complication"). Hypoxaemia was defined as oxygen saturations less than $80 \%$ and hypotension as any new requirement for vasopressors. Intra-operative bleeding was defined as bleeding that was severe enough to require conversion from PDT to ST and/or injury to great vessels at open procedure and/or requirement for intra-operative blood transfusion. Post-operative bleeding was defined as bleeding that required blood transfusion or surgical exploration. Where one complication occurred as a result of another (e.g. hypoxia as a result of bleeding, or loss of 
airway), then only the primary complication was recorded. Peri-operative change in $\mathrm{PaO}_{2} / \mathrm{FiO}_{2}$ ratio was defined as the change in $\mathrm{PaO}_{2} / \mathrm{FiO}_{2}$ ratio from the pre-operative value, to that recorded $24 \mathrm{~h}$ post-operatively.

\section{Outcome measures}

The primary outcome measure was the occurrence of any peri-operative complication. Secondary outcome measures were the peri-operative change in $\mathrm{PaO}_{2} / \mathrm{FiO}_{2}$ ratio, time to liberation from mechanical ventilation following tracheostomy, time to decannulation following tracheostomy, and death during the follow-up period.

\section{Results}

Between 1st March and 5th May 2020, 201 patients were admitted to an ICU in one of our hospitals with SARSCoV-2 infection requiring mechanical ventilation and subsequent tracheostomy. Detailed patient characteristics are shown in Table 1. PDT and ST groups demonstrated similar baseline characteristics, APACHE II score $(p=0.75)$ and lowest $\mathrm{PaO}_{2} / \mathrm{FiO}_{2}$ ratio $(p=0.85)$ in first $24 \mathrm{~h}$ of mechanical ventilation. With a median follow-up of 73 days, a total of 29 patients died after tracheostomy insertion irrespective of the insertion technique (14.4\%). Median [IQR(range)] duration of mechanical ventilation prior to tracheostomy was 17 [13.0-21.0 (0-44)] days, with no difference between groups $(p=0.53) . \mathrm{PaO}_{2} / \mathrm{FiO}_{2}$ ratio and coagulation parameters on the day of tracheostomy were also similar between groups.
Use of therapeutic doses of anticoagulation (either therapeutic low-molecular-weight heparin, systemic heparin or argatroban) was similar between the groups $(p=0.31)$.

PDT was performed in $124(62 \%)$ of patients, the vast majority of which were performed at the bedside in the ICU. Ninety-two of these $(74 \%)$ were performed by the ICU team, with the remainder being performed by either an otorhinolaryngologist or oral maxillofacial surgeon. ST was performed in 77 (38\%) of patients. The majority of ST were performed in the operating theatre, but seven of these were performed at the bedside in the ICU.

\section{Primary outcome: peri-operative complications}

Peri-operative complications occurred in 38 (18.9\%) of the whole cohort with no difference between PDT and ST ( $p=0.46$, Table 2). Intra-operative complications occurred in $6.5 \%$ of our cohort, with no difference between PDT and ST $(p=0.77)$. Amongst the 124 patients in the PDT group, intra-operative complications included bleeding $(n=1)$, cuff rupture $(n=1)$, hypoxia $(n=1)$, misplacement $(n=2)$, tracheal injury $(n=3)$ and loss of airway $(n=1)$. The only intra-operative complication reported in the ST group was hypoxia $(n=4)$. Post-operative complications occurred in $12.4 \%$ of the cohort, with no difference between PDT and ST $(p=0.19)$. In the PDT group, post-operative complications included bleeding $(n=8)$, dislodgement $(n=3)$ and pneumothorax $(n=1)$. In the ST group, post-operative complications included bleeding $(n=8)$, cuff leak $(n=1)$, dislodgement $(n=1)$ and hypoxia $(n=3)$. There were no significant differences in specific intra-operative or post-operative

Table 1 Characteristics of patients undergoing surgical or percutaneous tracheostomy formation for weaning from mechanical ventilation. Values presented as mean (SD), median (IQR [range]) or number (proportion)

\begin{tabular}{|c|c|c|c|c|}
\hline Characteristic & All patients $(n=201)$ & $\begin{array}{l}\text { Percutaneous tracheostomy } \\
(n=124)\end{array}$ & Surgical tracheostomy $(n=77)$ & $p$ value \\
\hline Age & $55.6(11.2)$ & $56.0(11.1)$ & $55.0(11.4)$ & 0.56 \\
\hline Female & $59(29.4 \%)$ & $38(30.6 \%)$ & $21(27.3 \%)$ & 0.64 \\
\hline BMI & $29.7(6.6)$ & $28.9(5.5)$ & $31.1(7.8)$ & 0.13 \\
\hline APACHE II & $14.0[12.0-17.0(4.0-26.0)]$ & $14.0[12.0-17.0(4.0-22.0)]$ & $15.0[12.0-17.0(6.0-26.0)]$ & 0.75 \\
\hline $\begin{array}{l}\text { Lowest PF ratio in first } 24 \mathrm{~h} \\
\text { of mechanical ventilation } \\
(\mathrm{mmHg})\end{array}$ & 114.5 [85.8-161.4 (28.3-520.8)] & $\begin{array}{l}116.5[87.3-160.8(28.3- \\
337.5)]\end{array}$ & $\begin{array}{l}111.4[78.0-164.5(39.5- \\
520.8)]\end{array}$ & 0.85 \\
\hline $\begin{array}{l}\text { Duration of mechanical ventila- } \\
\text { tion prior to tracheostomy (d) }\end{array}$ & $17.0[13.0-21.0(0-44)]$ & $16.0[13.0-22.0(3-44)]$ & $17.0[14.0-21.0(0-42)]$ & 0.53 \\
\hline $\begin{array}{l}\text { PF ratio on day of tracheostomy } \\
(\mathrm{mmHg})\end{array}$ & $\begin{array}{l}200.6[167.1-253.2(63.8- \\
857.2)]\end{array}$ & $\begin{array}{l}202.1[167.3-245.6(77.8- \\
857.2)]\end{array}$ & $\begin{array}{l}196.9[167.8-255.8(63.8- \\
461.3)]\end{array}$ & 0.78 \\
\hline $\begin{array}{l}\text { Platelet count on day of trache- } \\
\text { ostomy }\end{array}$ & $\begin{array}{l}287.0[206.0-409.0(21.0- \\
726.0)]\end{array}$ & $\begin{array}{l}312.5[211.5-436.5(21.0- \\
\quad 726.0)]\end{array}$ & $\begin{array}{l}267.0[199.0-364.0(64.0- \\
588.0)]\end{array}$ & 0.06 \\
\hline INR on day of tracheostomy & $1.1[1.0-1.2(0.9-3.6)]$ & $1.1[1.0-1.2(0.9-1.5)]$ & $1.1[1.0-1.2(0.9-3.6)]$ & 0.53 \\
\hline $\begin{array}{l}\text { Therapeutic anticoagulation on } \\
\text { day of tracheostomy }\end{array}$ & $92.0(45.8 \%)$ & $53.0(42.7 \%)$ & $39.0(50.6 \%)$ & 0.31 \\
\hline
\end{tabular}

APACHE II acute physiology and chronic health evaluation II, PF ratio $\mathrm{PaO}_{2} / \mathrm{FiO}_{2}$ ratio, INR international normalised ratio 
Table 2 Outcomes of patients undergoing percutaneous or surgical tracheostomy

\begin{tabular}{|c|c|c|c|c|}
\hline Outcome & All patients $(n=201)$ & $\begin{array}{l}\text { Percutaneous tracheostomy } \\
(n=124)\end{array}$ & Surgical tracheostomy $(n=77)$ & $p$ value \\
\hline $\begin{array}{l}\text { Total peri-operative complica- } \\
\text { tions }\end{array}$ & $38(18.9 \%)$ & $21(16.9 \%)$ & $17(22.1 \%)$ & 0.46 \\
\hline Intra-operative complications & $13(6.5 \%)$ & $9(7.3 \%)$ & $4(5.2 \%)$ & 0.77 \\
\hline Bleeding & 1 & 1 & 0 & \\
\hline Cuff rupture & 1 & 1 & 0 & \\
\hline Hypoxia & 5 & 1 & 4 & \\
\hline Misplacement & 2 & 2 & 0 & \\
\hline Tracheal injury & 3 & 3 & 0 & \\
\hline Loss of airway & 1 & 1 & 0 & \\
\hline Post-operative complications & $25(12.4 \%)$ & $12(9.7 \%)$ & $13(16.9 \%)$ & 0.19 \\
\hline Bleeding & 16 & 8 & 8 & \\
\hline Cuff leak & 1 & 0 & 1 & \\
\hline Dislodgement & 4 & 3 & 1 & \\
\hline Hypoxia & 3 & 0 & 3 & \\
\hline Pneumothorax & 1 & 1 & 0 & \\
\hline $\begin{array}{l}\text { Peri-operative change in } \mathrm{PF} \\
\text { ratio }(\mathrm{mmHg})\end{array}$ & $\begin{array}{l}2.0[-37.4-35.1(-328.6- \\
540.4)]\end{array}$ & $\begin{array}{l}5.5[-29.3-34.8(-328.6- \\
540.4)]\end{array}$ & $\begin{array}{l}-2.1[-67.1-32.4(-253.2- \\
130.8)]\end{array}$ & 0.08 \\
\hline $\begin{array}{l}\text { Time from tracheostomy } \\
\text { insertion to liberation from } \\
\text { mechanical ventilation (days) }\end{array}$ & $14.0[9.0-23.0(1.0-62.0)]$ & $14.0[9.0-21.5(1.0-62.0)]$ & $14.0[8.0-26.0(1.0-61.0)]$ & 0.93 \\
\hline $\begin{array}{l}\text { Time from tracheostomy inser- } \\
\text { tion to decannulation (das) }\end{array}$ & $20.0[15.0-31.5(5.0-70.0)]$ & $19.0[15.0-30.2(5.0-65.0)]$ & $21.0[15.5-36.0(5.0-70.0)]$ & 0.49 \\
\hline Death during follow-up period & $29.0(14.4 \%)$ & $17(13.7 \%)$ & $12(15.6 \%)$ & 0.84 \\
\hline
\end{tabular}

Values presented as median [IQR (range)], or number (proportion)

PF ratio $\mathrm{PaO}_{2} / \mathrm{FiO}_{2}$ ratio

complications between techniques. There was no difference in the rate of peri-operative bleeding between patients who were therapeutically anticoagulated and those that were not $(9(10.8 \%)$ vs. $8(7.9 \%), p=0.61)$.

\section{Secondary outcomes}

The median [IQR(range)] peri-operative change in $\mathrm{PaO}_{2} /$ $\mathrm{FiO}_{2}$ ratio in the PDT group was + 5.5 [-29.3-34.8 (-328.6-540.4)] mmHg, compared to -2.1 [ $-67.1-32.4$ [-253.2-130.8)] mmHg in the ST group, which approached significance ( $p=0.08$, Table 2). Median [IQR(range)] time to liberation from mechanical ventilation and decannulation following tracheostomy insertion was 14 [9.0-23.0 (1.0-62.0)] days and 20 [15.0-31.5 (5.0-70.0)] days, respectively, with no difference between PDT and ST $(p=0.93, p=0.49$, Figs. 1 and 2). Overall mortality was $14.4 \%$, with no difference between groups $(p=0.84)$. Two patients died as a result of a tracheostomy-related complication, one following a pneumothorax and one following a blocked tracheostomy tube as a result of post-operative bleeding. Remaining deaths were related to complications of COVID-19. Twenty-six patients died prior to tracheostomy decannulation, and one death occurred following decannulation on the ward. Of the 172 patients that were alive at follow-up, two remained ventilated in our hospitals and 163 were successfully decannulated. Seven patients were transferred to other hospitals ventilated, or discharged home with missing decannulation data.

\section{Discussion}

To our knowledge, this is the largest multicentre comparison of PDT and ST in patients with COVID-19 pneumonitis. Our groups had comparable pre-tracheostomy characteristics and we found no difference in the rates of either intra-operative or post-operative complications, or time to decannulation between the two groups. Previous studies and meta-analyses comparing complication rates between PDT and ST in nonCOVID-19 patients have reported variable results, although some describe a trend towards decreased rates of bleeding and wound infection when performing PDT [6-15]. Within the PDT group in our analysis, there were three tracheal injuries and two misplacements, whereas none were reported in the ST group. However, none of these complications were 

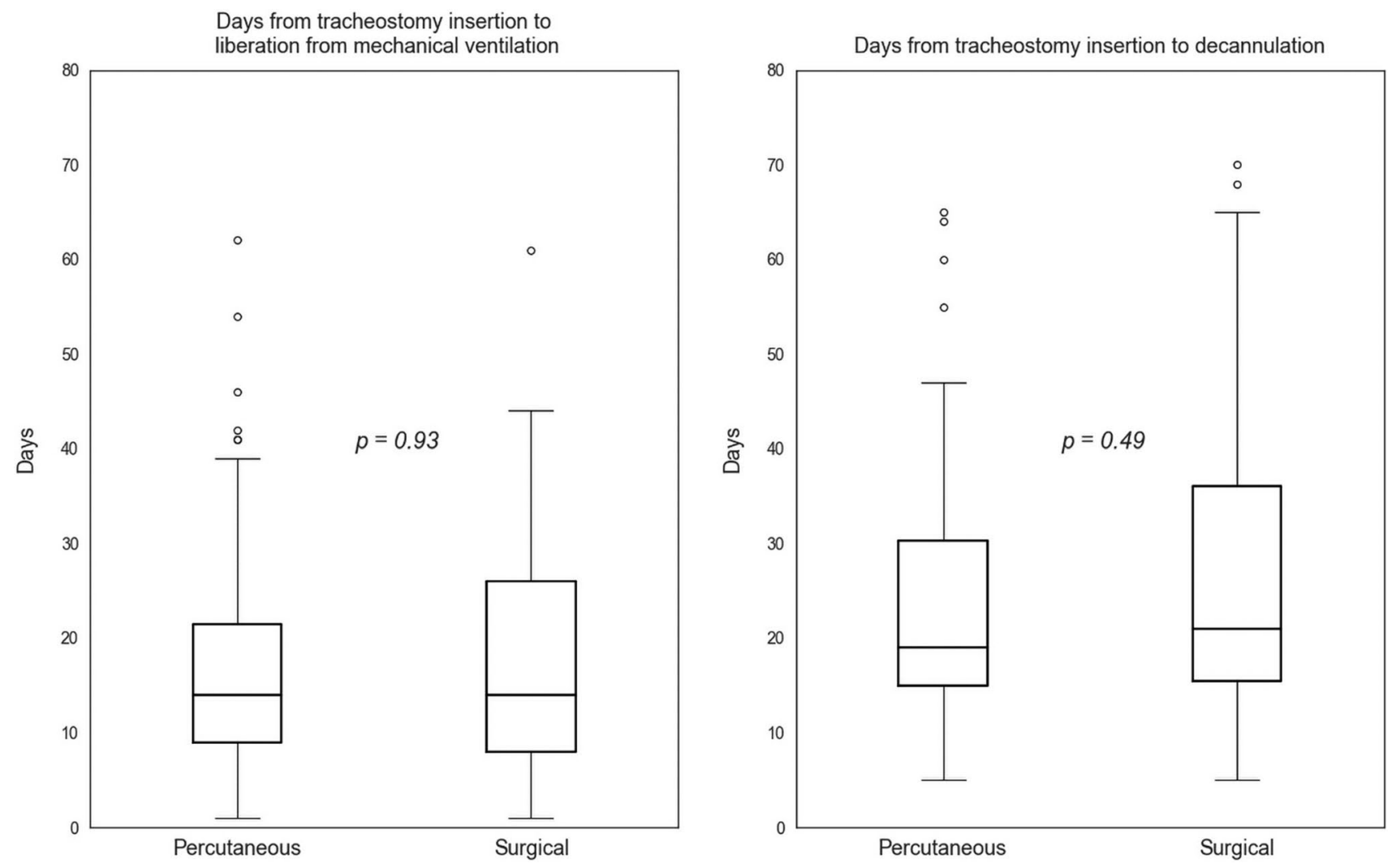

Fig. 1 Time to liberation from mechanical ventilation and tracheostomy decannulation. Median+IQR represented by boxes. Whiskers $1.5 \times \mathrm{IQR}$. Significant outliers marked

life threatening or affected patient outcomes. Increased incidence of 'technical difficulties' with PDT compared to ST has been described in the non-COVID-19 literature, although these are variably defined [15].

Both intra- and post-operative complication rates for PDT and ST were comparable to those in the non-COVID-19 literature $[6-15,18]$. Bleeding was the most common clinically significant post-operative complication in our cohort, occurring in $8.5 \%$ of our patients. Patients with COVID-19 exhibit a prothrombotic state [19], and a large proportion of our cohort (46\%) were receiving therapeutic anticoagulation in the peri-operative period. Our bleeding rates are comparable to those in the non-COVID-19 literature, suggesting that our approach to anticoagulation and tracheostomy does not confer an increased peri-operative bleeding risk.

The ST group showed a greater incidence of intra-operative hypoxia compared to the PDT group. In addition, the ST group showed a decrease in peri-operative $\mathrm{PaO}_{2} /$ $\mathrm{FiO}_{2}$ ratio whereas the PDT group showed an increase, with trend towards significance, warranting further investigation. $\mathrm{PaO}_{2} / \mathrm{FiO}_{2}$ ratio is commonly used as a marker of oxygenation [20], and there are a number of reasons why oxygenation might deteriorate during ST. The vast majority of patients undergoing ST were transferred from the ICU to the operating theatre on a portable ventilator, which in itself can destabilise patients with difficult oxygenation. Multiple ventilator-patient disconnections are usually required when patients are transferred from the ICU to the operating room, and although this was avoided where possible in COVID-19 patients to minimise to the risk of aerosolization, this may have led to alveolar de-recruitment. ST usually takes longer than PDT [15] and prolonged supine positioning on the operating table may de-recruit vulnerable alveoli in COVID-19 pneumonitis [21]. Numbers are too small to make any definitive conclusions, and the clinical significance of these findings is questionable.

Whilst we did not include specific indications for ST in our analysis, ST might be necessary, for example, when the front-of-neck anatomy is unfavourable or where there is poor neck extension. Decision to perform either PDT or ST in our centres, however, was largely a pragmatic one, based on local availability of resource and expertise during a period that placed huge pressure on critical care services. PDT is a more time-efficient procedure when compared with ST [15], and although this was not included in our analysis, this is an important consideration during a surge in activity where demands on tracheostomy services are high. In addition, one of our centres was able to rapidly train both 


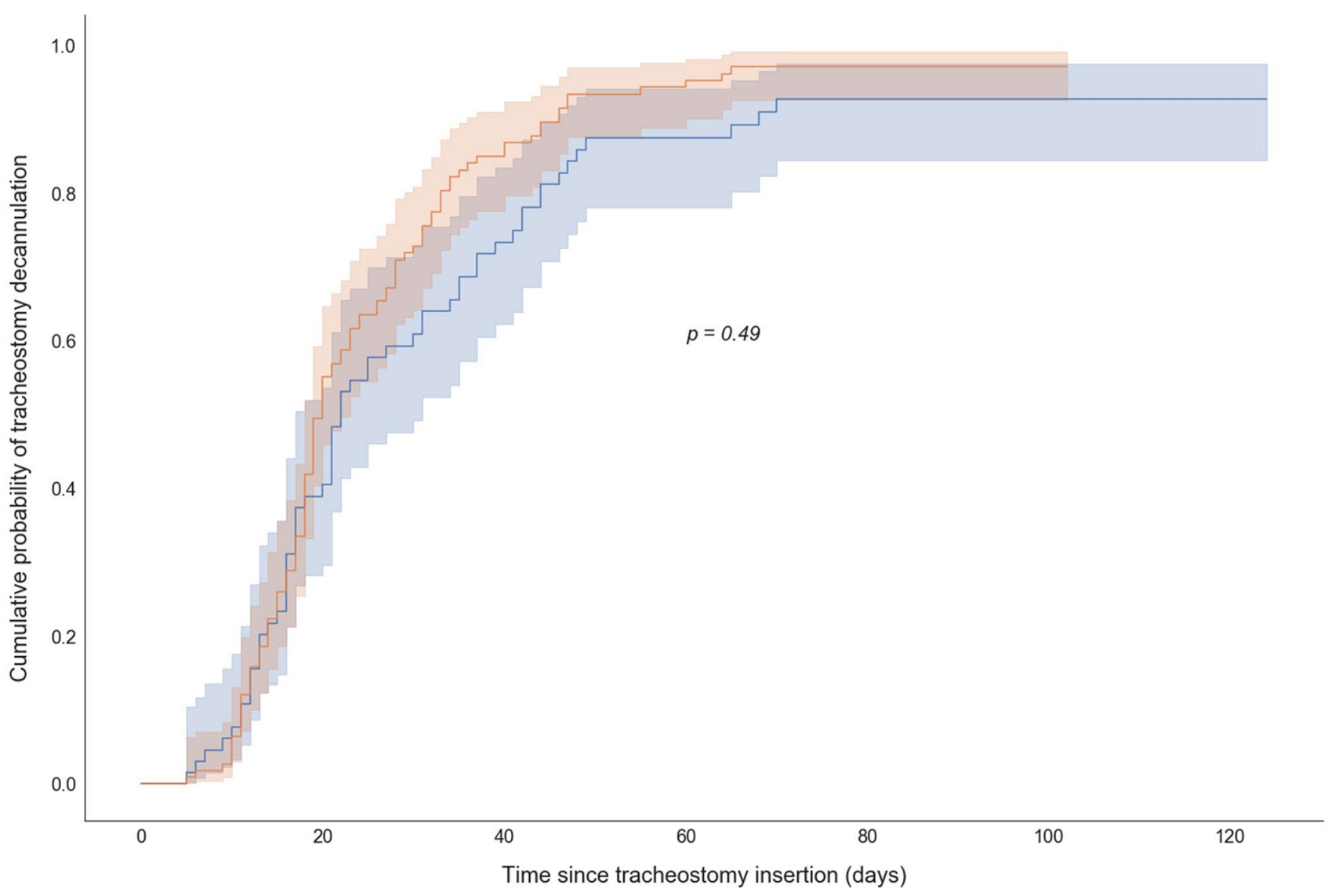

Fig. 2 Kaplan-Meier cumulative probability of tracheostomy decannulation in survivors including 95\% confidence interval plotted to day of last follow-up. (---) surgical, (---) percutaneous

otorhinolaryngologists and oral maxillofacial surgeons to perform PDT safely [17]. On the other hand, some centres were limited by supplies of PDT insertion kits due to widespread shortages, and having a flexible approach to the choice of tracheostomy formation technique in these situations has clear advantages.

Liberation from mechanical ventilation is a crucial milestone for patients who survive a prolonged critical care admission, and we have shown that a multidisciplinary approach to tracheostomy insertion, ventilatory weaning and decannulation can result in excellent outcomes for patients with COVID-19 [22]. In our study, we have shown that this is independent of the choice of tracheostomy insertion technique.

There are several potential limitations to this analysis that warrant discussion. During the COVID-19 pandemic, large numbers of healthcare professionals were redeployed to support critical care and although appropriate training was delivered, both the operators performing the tracheostomy, and the multidisciplinary healthcare professionals involved in post-tracheostomy care were relatively inexperienced. This may have affected the incidence of peri-operative complications. However, the potential effects of this confounder would affect both techniques equally. Although definitions for complications were strict at the moment of data collection, interpretation and possible bias may have affected the final results. Finally, although this is the largest multicentre comparison of PDT and ST in patients with COVID19-related respiratory failure undergoing tracheostomy, the number of reported complications is low, and further investigation in a large randomised control trial is required.

\section{Conclusion}

In patients with COVID-19 pneumonitis that required tracheostomy to facilitate weaning from mechanical ventilation, there was no difference in outcomes between those patients that had PDT compared with those that had ST. Planning for future surges in COVID-19-related critical care demands should embrace a pragmatic multidisciplinary approach to tracheostomy formation, incorporating all available resource and expertise. 
Acknowledgements The authors would like to thank J. Clarke and E. Scott for their contributions to this study. No external funding and no competing interests declared.

\section{Compliance with ethical standards}

Conflicts of interest The authors do not have any conflict of interest.

Human participants and/or animals Using the NHS Health Research Authority decision tool this project was determined to be a service evaluation thereby not requiring ethical approval, and was registered with the audit departments of each participating hospital. Principles of Good Clinical Practice were adhered to throughout the study.

\section{References}

1. Intensive care national audit and research centre. COVID-19 report. Available from: https://www.icnarc.org/Our-Audit/Audit s/Cmp/Reports. Accessed 15 Sept 2020

2. Griffiths J, Barber VS, Morgan L, Young JD (2005) Systematic review and meta-analysis of studies of the timing of tracheostomy in adult patients undergoing artificial ventilation. BMJ 330:1243-1246

3. Young D, Harrison DA, Cuthbertson BH, Rowan K (2013) Effect of early vs late tracheostomy placement on survival in patients receiving mechanical ventilation: the TracMan randomized trial. JAMA 309:2121-2129

4. Siempos II, Ntaidou TK, Filippidis FT, Choi AMK (2015) Effect of early versus late or no tracheostomy on mortality and pneumonia of critically ill patients receiving mechanical ventilation: a systematic review and meta-analysis. Lancet Respir Med 3:150-158

5. Nieszkowska A, Combes A, Luyt C-E et al (2005) Impact of tracheotomy on sedative administration, sedation level, and comfort of mechanically ventilated intensive care unit patients. Crit Care Med 33:2527-2533

6. Dulguerov P, Gysin C, Perneger TV, Chevrolet JC (1999) Percutaneous or surgical tracheostomy: a meta-analysis. Crit Care Med 27:1617-1625

7. Delaney A, Bagshaw SM, Nalos M (2006) Percutaneous dilatational tracheostomy versus surgical tracheostomy in critically ill patients: a systematic review and meta-analysis. Crit Care 10:R55

8. Pappas S, Maragoudakis P, Vlastarakos P et al (2011) Surgical versus percutaneous tracheostomy: an evidence-based approach. Eur Arch Otorhinolaryngol 268:323-330
9. Silvester W, Goldsmith D, Uchino S et al (2006) Percutaneous versus surgical tracheostomy: a randomized controlled study with long-term follow-up. Crit Care Med 34:2145-2152

10. Oliver ER, Gist A, Gillespie MB (2007) Percutaneous versus surgical tracheotomy: an updated meta-analysis. Laryngoscope 117:1570-1575

11. Brass P, Hellmich M, Ladra A, Ladra J, Wrzosek A (2016) Percutaneous techniques versus surgical techniques for tracheostomy (review). Cochrane Database Syst Rev 7:CD008045

12. Freeman BD, Isabella K, Lin N, Buchman TG (2000) A metaanalysis of prospective trials comparing percutaneous and surgical tracheostomy in critically ill patients. Chest 118:1412-1418

13. Higgins KM, Punthakee X (2007) Meta-analysis comparison of open versus percutaneous tracheostomy. Laryngoscope 117:447-454

14. Cheng E, Fee WE (2000) Dilatational versus standard tracheostomy: a meta-analysis. Ann Otol Rhinol Laryngol 109:803-807

15. Putensen $\mathrm{C}$, Theuerkauf N, Guenther U, Vargas M, Pelosi P (2014) Percutaneous and surgical tracheostomy in critically ill adult patients: a meta-analysis. Crit Care 18:544

16. Chiesa-Estomba CM, Lechien JR, Calvo-Henríquez C, et al. (2020) Systematic review of international guidelines for tracheostomy in COVID-19 patients. Oral Oncol 108:104844

17. Takhar A, Walker A, Tricklebank S et al (2020) Recommendation of a practical guideline for safe tracheostomy during the COVID19 pandemic. Eur Arch Otorhinolaryngol 277:2173-2184

18. Lipton G, Stewart M, McDermid R et al (2020) Multispecialty tracheostomy experience. Ann R Coll Surg Engl 102:343-347

19. Levi M, Thachil J, Iba T, Levy JH (2020) Coagulation abnormalities and thrombosis in patients with COVID-19. Lancet Haematol 7:e438-e440

20. The ARDS definition task force (2012) Acute respiratory distress syndrome: the Berlin definition. JAMA 307:2526-2533

21. Marini JJ, Gattinoni L (2020) Management of COVID-19 respiratory distress. JAMA 323:2329-2330

22. Rovira A, Dawson D, Walker A et al (2020) Tracheostomy care and decannulation during the COVID-19 pandemic. A multidisciplinary clinical practice guideline. Eur Arch Oto-Rhino-Laryngol. https://doi.org/10.1007/s00405-020-06126-0 (Published online ahead of print)

Publisher's Note Springer Nature remains neutral with regard to jurisdictional claims in published maps and institutional affiliations.

\section{Affiliations}

\section{Aleix Rovira ${ }^{1}\left[\right.$ - Stephen Tricklebank ${ }^{2}$ - Pavol Surda ${ }^{1}$ - Stephen Whebell ${ }^{2}$. Joe Zhang ${ }^{2}$. Arun Takhar ${ }^{1}$. Elizabeth Yeung ${ }^{3} \cdot$ Kathleen Fan $^{3} \cdot$ Imran Ahmed $^{4}$. Phillip Hopkins ${ }^{5}$. Deborah Dawson ${ }^{6}$. Jonathan Ball ${ }^{6}$. Ram Kumar $^{7} \cdot$ Waqas Khaliq $^{8} \cdot$ Ricard Simo $^{1} \cdot$ Asit Arora $^{1}$}

1 Department of Otolaryngology and Head and Neck Surgery, Guy's and St Thomas' NHS Foundation Trust, Great Maze Pond, London SE1 9RT, UK

2 Department of Critical Care, Guy's and St Thomas' NHS Foundation Trust, London, UK

3 Department of Oral and Maxillofacial Surgery, Kings College Hospital NHS Foundation Trust, London, UK
4 Department of Anaesthesia, Guy's and St Thomas' NHS Foundation Trust, London, UK

5 Department of Critical Care, Kings College Hospital NHS Foundation Trust, London, UK

6 Department of Critical Care, St George's University Hospitals NHS Foundation Trust, London, UK 
7 Department of Critical Care, Kingston Hospital NHS Foundation Trust, London, UK
8 Department of Critical Care, Lewisham and Greenwich NHS Trust, London, UK 